\title{
Wall parameters estimation based on support vector regression for through wall radar sensing
}

\author{
Xi Chen ${ }^{1,2}$ and Weidong Chen ${ }^{1,2^{*}}$
}

\begin{abstract}
In through wall radar sensing, the wall parameters estimation (WPE) problem has been a topic that attracts a lot of attention since the wall parameters, i.e., the permittivity and the thickness, are of crucial importance to locate the targets and to produce a well-focused image, but they are usually unknown in practice. To solve this problem, in this paper, the support vector regression (SVR), a powerful tool for regression analysis, is introduced, and its performance on WPE, provided it is used it in the regular way, is investigated. Unfortunately, it is shown that the regular use of SVR cannot afford satisfactory estimation results since the sample data used in SVR, namely the received echoes from the walls, are seriously interfered with the echoes from the targets which are located near the walls. In view of this limitation, a novel SVR-based WPE approach that consists of three stages is proposed by this paper. In the first stage, three regression functions are trained by SVR, one of which will output the estimate of the permittivity in the second stage, and the others are designed to output two instrumental variables for estimating the thickness. In the third stage, the estimate of thickness will be achieved by minimizing a predefined cost function wherein the estimated permittivity and the outputted instrumental variables are involved. The better robustness and higher estimation accuracy of the proposed approach compared to the regular use of SVR are validated by the numerical experimental results using finite-difference time-domain simulations.
\end{abstract}

Keywords: Through-wall radar sensing; Wall parameters; Parameter estimation; Support vector regression; Cost function

\section{Introduction}

Through-wall radar sensing (TWRS) has been a research field of great interest in recent years due to its capability of remotely surveying the contents behind an opaque wall [1-4]. Nevertheless, compared with the signal propagation in free space, the radar returns passing through the wall will be further attenuated and delayed. As a result, the detectability of the targets decreases, and the target images are displaced and defocused [5-7]. In order to alleviate such performance degradations, the extra attenuation and delay due to the wall should be compensated appropriately in the post processing under the general

\footnotetext{
*Correspondence: wdchen@ustc.edu.cn

${ }^{1}$ Key Laboratory of Electromagnetic Space Information, Chinese Academy of Sciences, Huangshan Road, 230027 Hefei, People's Republic of China

${ }^{2}$ Department of Electronic Engineering and Information Science, University of Science and Technology of China, Huangshan Road, 230027 Hefei, People's Republic of China
}

assumption that the wall parameters are known a priori. However, this kind of assumption is not usually available for most TWRS applications in practice. Furthermore, measuring the wall parameters using electronic measurement equipment, which needs access to both sides of the wall, is not feasible in most scenarios $[8,9]$.

To estimate the wall parameters, some methods have been developed and they can be roughly classified into three main categories. The first one using multiple measurements with diverse array structures or at different standoff distances is complicated and hard to implement in reality $[10,11]$. The second one refers to the autofocusing techniques, which use iterative optimization schemes and thus are computationally expensive and time consuming $[12,13]$. The last one searching the parameters by maximizing the correlation between the simulated radar return derived from a pre-established parametric model, and the real radar return may have unexpected errors

\section{Springer}

(c) 2015 Chen and Chen. This is an Open Access article distributed under the terms of the Creative Commons Attribution License (http://creativecommons.org/licenses/by/4.0), which permits unrestricted use, distribution, and reproduction in any medium provided the original work is properly credited. 
since the model may not exactly coincide with the actual environment [14].

Differing from the common methods above, this paper provides a new idea that brings the support vector regression (SVR) into the wall parameter estimation (WPE) problem. As an extended version of support vector machine (SVM), SVR is formulated for the case of regression while SVM is usually used for classification and recognition. Originally, SVM is introduced to identify and classify the targets from radar images and high-resolution range profiles [15-17], and then applied into more applications, e.g., ground-penetrating radar (GPR) and ocean clutter suppression [18-20]. Recently, SVR has also been involved in radar areas to solve regression problems, such as prediction of the vehicle travel time [21] and parameter estimation in GPR [22-24]. Inspired by these successful applications, this paper brings SVR into TWRS to estimate the wall parameters and uses it in the regular way, which can be noted as the regular SVR method.

Nevertheless, applying the regular SVR method in WPE is not enough. The estimation results show that the regular SVR method cannot satisfy the requirements of WPE in accuracy and robustness, which we think is mainly because of two reasons. The first reason is that the radar returns used as training data are collected in a controlled laboratory environment and thus only contain the echoes coming from the walls, whereas in practice the received radar returns, i.e., test data may contain target echoes as well as wall echoes. The difference between the training and the test data is so significant that the regression functions established by the training data cannot work well for the test data, leading to the estimation performance deterioration. The second reason is that the regular SVR method training an independent regression function for each parameter, and estimating the parameter separately by its own function does not take into consideration the fact that the parameters are coupled with each other in the wall echoes. Therefore, the estimation result of one parameter is not utilized for estimating the other parameter, which means some valuable information that should be exploited are discarded in the regular SVR method.

Considering the above problems, in this paper, a new three-stage approach combining SVR and an optimization procedure is presented. In the first stage, three regression functions are trained by SVR based on the training data, i.e., the echoes coming from different walls. One of the functions is used to estimate the permittivity in the second stage when a radar return is received from an unknown wall, while the others outputting instrumental variables are designed for estimating the thickness. Subsequently in the last stage, estimation of the thickness will be implemented by minimizing a predefined cost function based on the estimated permittivity and the instrumental variables outputted from the other two regression functions.
In the proposed approach, SVR is expected to output the estimates of the permittivity and the instrumental variables in a short time once the regression functions are established. Then, an optimization procedure is employed to estimate the wall thickness robustly and accurately. By introducing the instrumental variables and the optimization procedure, this approach avoids the performance degradation caused by the presence of the targets and improves the estimation accuracy. The numerical experimental results using finite-difference time-domain (FDTD) simulations validate the effectiveness of the proposed approach.

This paper is organized as follows. Section 2 gives the TWRS signal model and a brief review of SVR. The regular SVR method used in WPE is presented in Section 3. In Section 4, the proposed SVR-based WPE approach is detailed. The simulation results of the proposed approach and the regular method are shown and compared in Section 5. Conclusions are drawn in Section 6.

\section{Essential backgrounds}

In this section, we give a brief description of the throughwall radar returns which are used as the sample data in SVR and a short review of the SVR to show how it works.

\subsection{TWRS signal model}

Consider that a region of interest behind a single-layered homogeneous wall is illuminated by a radar system at a certain standoff distance, in which the transmitted signal can be a short pulse with large bandwidth. Since the wall is usually formed of lossy mediums, the wall transmissivity is a function of signal frequency, thus the received radar return cannot be simplified as the superposition of multiple time-shifted and scaled transmitted signals $s(t)$ [25]. As shown in Fig. 1, due to the presence of the wall, the first two echoes in the received return are the reflections from the front and the rear side of the wall and are denoted as $y_{f}(t)$ and $y_{r}(t)$ respectively. Then the received radar return can be written as [26]

$$
\begin{aligned}
z(t)= & y_{f}(t)+y_{r}(t)+\sum_{p=1}^{P} y_{p}(t)+n(t) \\
= & h_{f}(t) * s(t)+h_{r}(t) * s(t) \\
& +\sum_{p=1}^{P} h_{T}(t) * h_{p}(t) * s(t)+n(t)
\end{aligned}
$$

where $h_{f}(t)$ and $h_{r}(t)$ are the impulse responses of the front and the rear interface respectively. $h_{T}(t)$ is the twoway transmission response of the wall, and $h_{p}(t)$ denotes the response of the $p^{\text {th }}$ object in free space. $n(t)$ represents the additive Gaussian noise. 


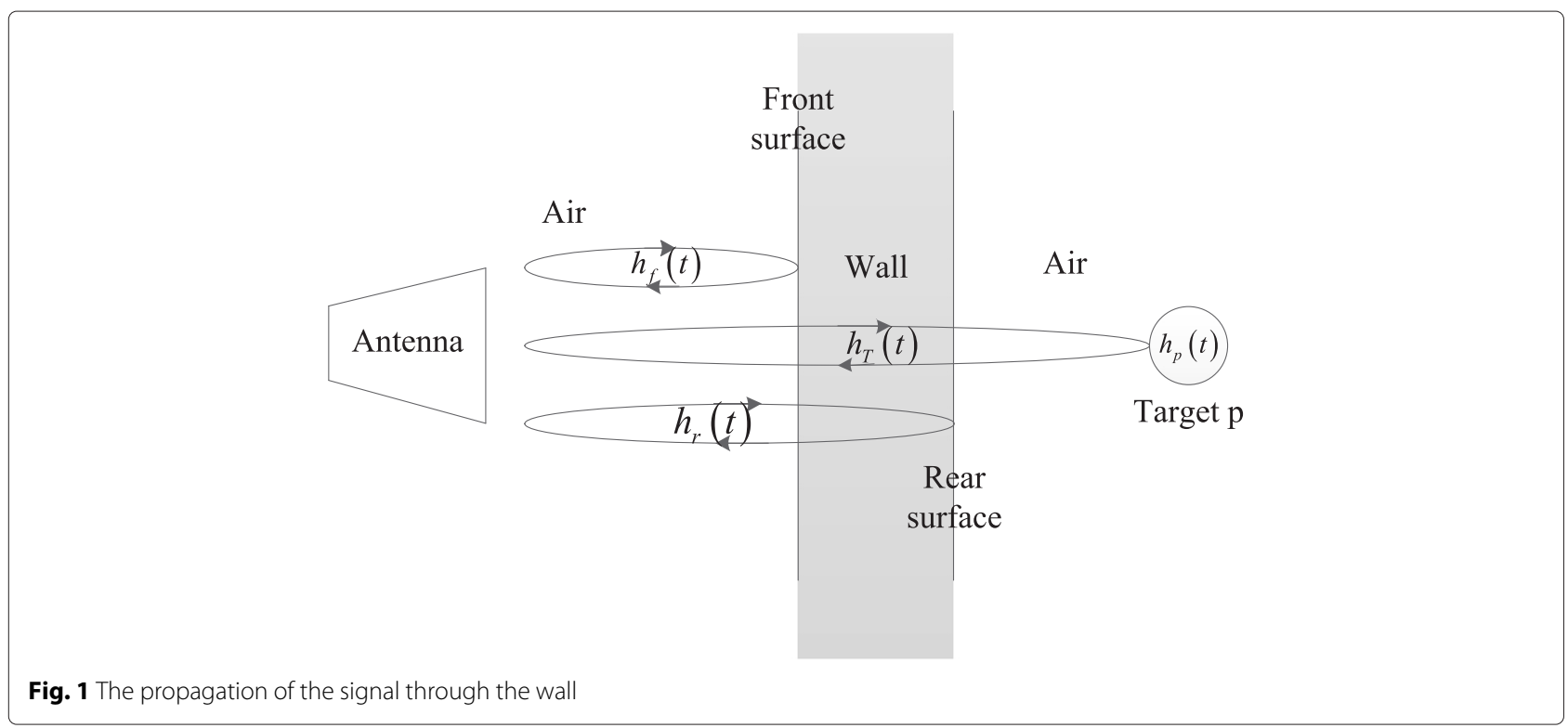

Assuming that the incident angle, the polarization mode, and the standoff distance are all fixed, under this situation, $h_{f}(t)$ can be considered to be only related to the permittivity of the wall since the wall can be viewed as a dielectric slab and, its permittivity can be considered constant within the bandwidth of the signal [27].

As for $h_{r}(t)$ and $h_{T}(t)$, they are associated with not only the permittivity, but also the wall thickness and the conductivity of the wall. The conductivity refers to the static electric conductivity of the wall and represents the ohmic loss. The imaginary part of the permittivity refers to the AC electric conductivity and represents the polarization loss. Since these two parameters are usually small and mainly affect the attenuation of the signal [28], they will not be estimated in this paper. Therefore, the wall parameters that we are focused on are the real part of the permittivity and the thickness.

\subsection{The principle of SVR}

Suppose that we are given training data

$$
\left\{\left(\mathbf{x}_{1}, y_{1}\right), \ldots,\left(\mathbf{x}_{l}, y_{l}\right)\right\} \subset \mathbb{R}^{n} \times \mathbb{R},
$$

where each $\mathbf{x}_{i}$ represents an input feature vector and has a corresponding target value $y_{i}$ for $i=1, \ldots, l$, where $l$ represents the size of the training data set. The basic idea of SVR is to find a function $f(x)$ that has at most $\varepsilon$ deviation from the actually obtained target values for all the training samples by mapping the training data from the input feature space into a higher dimensional space, and meanwhile has the capability to approximate future values as accurately as possible $[29,30]$. The linear function of SVR takes the form $f(\mathbf{x})=\langle\boldsymbol{\omega}, \mathbf{x}\rangle+b$ with $\omega \in \mathbb{R}^{n}, b \in \mathbb{R}$, where $\langle\cdot, \cdot\rangle$ denotes the dot product in $\mathbb{R}^{n}$.
In standard $\varepsilon$-SVR, the value of $\omega$ and $b$ can be determined as the solution of the following convex optimization problem

$$
\begin{aligned}
\min & \frac{1}{2}\|\boldsymbol{\omega}\|^{2}+C \sum_{i=1}^{l}\left(\xi_{i}+\xi_{i}^{*}\right) \\
\text { s.t. } & \left\{\begin{array}{c}
y_{i}-\left\langle\boldsymbol{\omega}, \mathbf{x}_{i}\right\rangle-b \leq \varepsilon+\xi_{i} \\
\left\langle\boldsymbol{\omega}, \mathbf{x}_{i}\right\rangle+b-y_{i} \leq \varepsilon+\xi_{i}^{*} \\
\xi_{i}, \xi_{i}^{*} \geq 0
\end{array}\right.
\end{aligned}
$$

where $C>0$ is a regularization constant, $\varepsilon$ is the tube radius, and $\xi_{i}$ and $\xi_{i}^{*}$ are the slack variables [31]. The vector $\omega$ can be written in terms of data points by solving the dual problem as

$$
\boldsymbol{\omega}=\sum_{i=1}^{l}\left(\alpha_{i}-\alpha_{i}^{*}\right) \mathbf{x}_{i}
$$

thus

$$
f(\mathbf{x})=\sum_{i=1}^{l}\left(\alpha_{i}-\alpha_{i}^{*}\right)\left\langle\mathbf{x}_{i}, \mathbf{x}\right\rangle+b
$$

where $\alpha_{i}$ and $\alpha_{i}^{*}$ are Lagrange multipliers.

For the variable $b$, it can be computed by applying the Karush-Kuhn-Tucker (KKT) conditions as

$$
\begin{aligned}
& b=y_{i}-\left\langle\boldsymbol{\omega}, \mathbf{x}_{i}\right\rangle-\varepsilon, \text { for } \alpha_{i} \in(0, C) \\
& b=y_{i}-\left\langle\boldsymbol{\omega}, \mathbf{x}_{i}\right\rangle+\varepsilon, \text { for } \alpha_{i}^{*} \in(0, C)
\end{aligned}
$$

The next step is to make the support vector algorithm nonlinear. Consider a nonlinear function $\Phi$ mapping the 
training data from $\mathbb{R}^{n}$ to a higher dimensional space. Thus Eq. (4) becomes

$$
\begin{aligned}
f(\mathbf{x}) & =\sum_{i=1}^{l}\left(\alpha_{i}-\alpha_{i}^{*}\right)\left\langle\Phi\left(\mathbf{x}_{i}\right), \Phi(\mathbf{x})\right\rangle+b \\
& =\sum_{i=1}^{l}\left(\alpha_{i}-\alpha_{i}^{*}\right) K\left(\mathbf{x}_{i}, \mathbf{x}\right)+b
\end{aligned}
$$

where $K\left(\mathbf{x}_{i}, \mathbf{x}\right)$ is the kernel function which enables the inner product in high dimensional space to be performed using low dimensional space data without knowing the transformation $\Phi$. Therefore, SVR can be run readily as long as the kernel function, the penalty $C$, and the radius $\varepsilon$ are chosen appropriately [32].

\section{The regular SVR method in WPE}

As stated above, through putting the feature vector into the regression function, we can estimate its target value once the regression function is established by SVR. Therefore, the radar returns from diverse walls with different parameters can be collected as the training data, and one can train a regression function for each of the wall parameters. Then, when we confront a new wall, its parameters can be estimated by taking its radar return into those corresponding regression functions.

Assuming that we have obtained a certain amount of radar returns from different walls, before the training phase, a procedure called feature extraction, which extracts the useful information from the data or transforms the data into an appropriate format, should be done to produce the feature vectors. The extracted feature vectors are deemed to be the better representations of the sample data and thus they are more suitable for the regression problem.

The feature vector $\mathbf{v}$ of the radar return extracted by the regular SVR method is produced as follows. The temporal radar return $z(t)$ can be transformed into frequency domain through

$$
\tilde{z}(f)=\mathcal{F} \mathcal{T}\{z(t)\}
$$

where $\mathcal{F} \mathcal{T}\{\cdot\}$ represents the Fourier transform of the associated temporal signal. For $N$ discrete frequencies within the bandwidth, the transformed signal $\tilde{z}(f)$ can be written in a vector form as

$$
\mathbf{z}=\left[\tilde{z}\left(f_{1}\right) \tilde{z}\left(f_{2}\right) \cdots \tilde{z}\left(f_{N}\right)\right]^{T} .
$$

The correlation matrix is defined as $\boldsymbol{\Gamma}=E\left[\mathbf{z z}^{H}\right]$, where $E[\cdot]$ is the expectation operator, and the superscript ${ }^{H}$ indicates the conjugate transpose. In practice, the correlation matrix $\Gamma$ is estimated from $M$ independent snapshots of the data vector $\mathbf{z}$

$$
\hat{\boldsymbol{\Gamma}}=\frac{1}{\mathrm{M}} \sum_{m=1}^{M} \mathbf{z}_{m} \mathbf{z}_{m}^{H} .
$$

The feature vector is then extracted from the correlation matrix [24]. Since the correlation matrix is Hermitian, only the upper triangular half of the matrix is used, and the elements in there are reorganized to form the feature vector $\mathbf{v}$ as follows:

$$
\begin{gathered}
\mathbf{v}=\left[r_{11}, \ldots, r_{N N}, \mathcal{R}\left(r_{12}\right), \mathcal{R}\left(r_{23}\right), \ldots, \mathcal{R}\left(r_{(N-1)(N)}\right),\right. \\
\mathcal{R}\left(r_{13}\right), \ldots, \mathcal{R}\left(r_{1 N}\right), \mathcal{I}\left(r_{12}\right), \mathcal{I}\left(r_{23}\right), \\
\left.\ldots, \mathcal{I}\left(r_{(N-1)(N)}\right), \mathcal{I}\left(r_{13}\right), \ldots, \mathcal{I}\left(r_{1 N}\right)\right]^{T}
\end{gathered}
$$

with $\mathcal{R}\left(r_{b c}\right)$ and $\mathcal{I}\left(r_{b c}\right)$ being the real and imaginary parts of $r_{b c}=[\boldsymbol{\Gamma}]_{b c}, c \geq b, b=1, \ldots, N$. Usually, the feature vector will be normalized before using SVR. In this paper, the feature vectors are all normalized with a scale between 0 to 1 .

In the regular way, the regression function is trained by SVR for each of the wall parameters independently. Thus there are two training data sets that need to be prepared for the two wall parameters. Let

$$
B_{\varepsilon_{r}}=\left\{\left(\mathbf{v}_{1}, \varepsilon_{r 1}\right), \ldots,\left(\mathbf{v}_{i}, \varepsilon_{r i}\right), \ldots,\left(\mathbf{v}_{l}, \varepsilon_{r l}\right)\right\},
$$

where $\mathbf{v}_{i}$ denotes the feature vector of the $i^{\text {th }}$ training sample and the permittivity $\varepsilon_{r i}$ is the corresponding target value. Similarly, Let

$$
B_{d}=\left\{\left(\mathbf{v}_{1}, d_{1}\right), \ldots,\left(\mathbf{v}_{i}, d_{i}\right), \ldots,\left(\mathbf{v}_{l}, d_{l}\right)\right\} .
$$

where $d_{i}$ used as target value is the thickness in the $i^{\text {th }}$ sample, and $l$ represents the number of the total training samples.

Following the instruction about SVR described above, taking the training data sets, $B_{\varepsilon_{r}}$ and $B_{d}$, into SVR individually, two regression functions will be obtained. This procedure is termed the training phase in machine learning. Then when a radar return from an unknown wall is received, we put its feature vector into the two regression functions to obtain the estimates of the permittivity and the thickness of the wall, respectively, and this step is called the test phase. For this application, it also can be termed as an estimation phase.

The schematic diagram of the regular SVR method is depicted in Fig. 2.

\section{The proposed SVR-based approach for WPE}

The proposed approach is comprised of three stages. In the first stage, the feature vectors are redesigned and extracted in a different way, and then three regression functions are trained. The estimation process is implemented in the last two stages. In the former, the permittivity of the wall is estimated by the regression function, and in the latter, the estimate of the thickness is obtained by minimizing a predefined cost function in which the estimated permittivity and the other two regression functions are involved. 


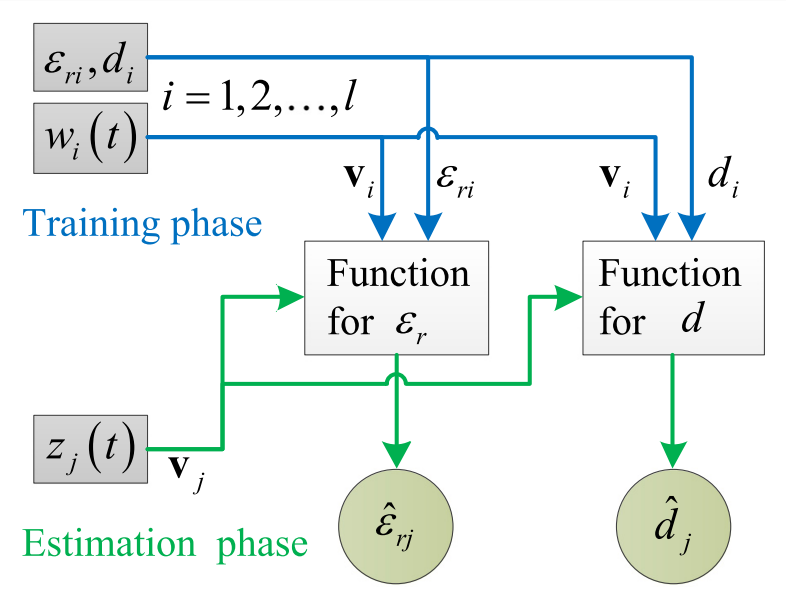

Fig. 2 Diagram of the regular SVR method

\subsection{The first stage}

It has been pointed out that the samples in training data set may be different from those in the test data set. In this situation, the regular SVR method introduced above cannot provide acceptable estimation results. Therefore, SVR ought to be used in a new way.

Suppose that the training data are given as

$$
\left\{w_{i}(t), \varepsilon_{i}, d_{i}\right\}, i=1,2, \ldots, l,
$$

where $w_{i}(t)$ is the radar return from the $i^{t h}$ wall with known parameters $\left(\varepsilon_{r i}, d_{i}\right)$ and can be written as

$$
w_{i}(t)=y_{f i}(t)+y_{r i}(t)+n_{i}(t) .
$$

These radar returns are usually collected in a laboratory in advance and do not contain target echoes. During the collection, the incident angle, polarization mode and the standoff distance are all set to be consistent.

Different from the regular SVR method, in order to meet the requirement of our approach, the feature extraction procedure is changed. Let $R_{i}(\tau)$ be the cross correlation function of $w_{i}(t)$ and the transmitted signal $s(t)$

$$
R_{i}(\tau)=\int w_{i}(t+\tau) s(t) d t
$$

In training data, since obviously the echoes from the front and the rear sides of the wall are dominant in the received signal, there is no doubt that the two highest peaks in $R_{i}(\tau)$ are derived from these two echoes, where the highest peak represents the echo from the front side while the second highest represents the echo from the rear side. Then, the magnitudes and time delays of these two peaks are extracted and denoted as $\left(\beta_{1 i}, \tau_{1 i}\right)$ and $\left(\beta_{2 i}, \tau_{2 i}\right)$, respectively.

As stated above, the echo from the front surface is only related to the permittivity of the wall. Therefore, for estimating the permittivity, we construct the training data set $B_{f}$ with $\mathbf{v}_{f i}$ being the feature vectors and $\varepsilon_{r i}$ being the target values, where

$$
\begin{aligned}
\mathbf{v}_{f i} & =\left[\beta_{1 i}, \tau_{1 i}\right]^{T} . \\
B_{f} & =\left\{\left(\mathbf{v}_{f 1}, \varepsilon_{r 1}\right), \ldots,\left(\mathbf{v}_{f i}, \varepsilon_{r i}\right), \ldots,\left(\mathbf{v}_{f l}, \varepsilon_{r l}\right)\right\} .
\end{aligned}
$$

Based on this training data set, the regression function of the permittivity is trained by SVR and noted as

$$
\varepsilon_{r}=g\left(\mathbf{v}_{f}\right)
$$

Then we select the parameters of the wall as another feature vector, that is

$$
\mathbf{v}_{s i}=\left[\varepsilon_{r i}, d_{i}\right]^{T} .
$$

These feature vectors are used to constitute two other training data sets, which are listed as

$$
\begin{aligned}
B_{\beta_{2}} & =\left\{\left(\mathbf{v}_{s 1}, \beta_{21}\right), \ldots,\left(\mathbf{v}_{s i}, \beta_{2 i}\right), \ldots,\left(\mathbf{v}_{s l}, \beta_{2 l}\right)\right\} . \\
B_{\tau_{2}} & =\left\{\left(\mathbf{v}_{s 1}, \tau_{21}\right), \ldots,\left(\mathbf{v}_{s i}, \tau_{2 i}\right), \ldots,\left(\mathbf{v}_{s l}, \tau_{2 l}\right)\right\} .
\end{aligned}
$$

These two sets are used to train the other two regression functions, which are noted as

$$
\begin{aligned}
\beta_{2} & =u\left(\mathbf{v}_{s}\right) \\
\tau_{2} & =v\left(\mathbf{v}_{s}\right),
\end{aligned}
$$

respectively. The instrumental variables outputted from these functions will be used to estimate the thickness of the wall later. For now, three regression functions have been trained and established.

\subsection{The second stage}

For the $j^{\text {th }}$ wall that need to be estimated, the received radar return from it is denoted as $z_{j}(t)$, which may contain the target's echoes furthermore, that is

$$
z_{j}(t)=y_{f j}(t)+y_{r j}(t)+\sum_{p=1}^{P} y_{p j}(t)+n_{j}(t)
$$

and its cross correlation function with the transmitted signal is given by

$$
R_{j}(\tau)=\int z_{j}(t+\tau) s(t) d t
$$

In $R_{j}$, the highest peak still represents the echo from the front side of the wall, but it is not sure if the second highest peak can stand for the echo from the rear side of the wall since this echo may be exceeded by the target echoes in amplitude. Even if the rear side echo has larger amplitude than the target echoes, the second highest peak cannot be viewed as the representation of the rear-side echo for sure since there is a possibility that the target echoes overlap with the rear side echo partly as long as the targets are located close to the wall. Therefore, the magnitude and the 
time delay of the second highest peak in $R_{j}$ are invalid and will not be extracted.

Let $\left(\beta_{1 j}, \tau_{1 j}\right)$ denote the magnitude and the time delay of the highest peak, which are the only features we extract from $R_{j}(\tau)$. We noticed that only $\beta_{1}$ and $\tau_{1}$ can be extracted from both the training and the test data. That is why we select them to constitute the feature vector $\mathbf{v}_{f}$.

Taking the feature vector $\mathbf{v}_{f j}$ into the function $g(\cdot)$, we have

$$
\hat{\varepsilon}_{r j}=g\left(\mathbf{v}_{f j}\right)
$$

This is the estimate of the permittivity of the $j^{\text {th }}$ wall.

\subsection{The third stage}

The task of the third stage is to estimate the thickness of the wall. Let us define a cost function at first, which is written as

$$
\begin{aligned}
C F\left(\mathbf{v}_{s}\right) & =\left\|R(\tau)-\beta_{2} \cdot R_{0}\left(\tau-\tau_{2}\right)\right\|_{1} \\
& =\left\|R(\tau)-u\left(\mathbf{v}_{s}\right) \cdot R_{0}\left(\tau-v\left(\mathbf{v}_{s}\right)\right)\right\|_{1}
\end{aligned}
$$

where $R_{0}(\tau)$ is the normalized autocorrelation function of $s(t),\|\cdot\|_{1}$ denotes the $L 1$ norm which is the integral of the absolute values of a function. In this equation, the other two regression functions $u\left(\mathbf{v}_{s}\right)$ and $v\left(\mathbf{v}_{s}\right)$ are used to provide the estimates of $\beta_{2}$ and $\tau_{2}$, respectively.

The motivation of creating this function is to measure the difference between the real echo from the wall rear side and a scaled and shifted transmitted signal, which can be expressed by Fig. 3. If the difference is small, it means that the instrumental variables $\beta_{2}$ and $\tau_{2}$ are estimated accurately by the regression functions which are associated with the wall parameters, and it further implies that at this moment thst the wall parameters used in the regression functions approximate to the true wall parameters. Therefore, the cost function will be a good indicator to the status of the estimation.

For the $j^{\text {th }}$ wall, since its estimated permittivity has been obtained, $\hat{\varepsilon}_{r j}$, we define

$$
\tilde{\mathbf{v}}_{s j}=\left[\hat{\varepsilon}_{r j}, d\right]^{T}
$$

where $d$ represents the thickness that has not been determined. Substituting $\tilde{\mathbf{v}}_{s j}$ for $\mathbf{v}_{s}$ in Eq. (26), we have

$$
\begin{aligned}
C F\left(\tilde{\mathbf{v}}_{s j}\right) & =\left\|R_{j}(\tau)-u\left(\tilde{\mathbf{v}}_{s j}\right) \cdot R_{0}\left(\tau-v\left(\tilde{\mathbf{v}}_{s j}\right)\right)\right\|_{1} \\
& =\left\|R_{j}(\tau)-u\left(\hat{\varepsilon}_{r j}, d\right) \cdot R_{0}\left(\tau-v\left(\hat{\varepsilon}_{r j}, d\right)\right)\right\|_{1}
\end{aligned}
$$

The estimate of the thickness is achieved by minimizing this cost function. Since $\hat{\varepsilon}_{r j}$ is known before, which implies that the cost function only has one variable, the estimate of the thickness can be expressed as follows,

$$
\begin{aligned}
& \hat{d}_{j}=\underset{d}{\arg \min } C F\left(\tilde{\mathbf{v}}_{s j}\right) \\
& =\underset{d}{\arg \min } C F_{j}\left(\hat{\varepsilon}_{r j}, d\right) \\
& =\underset{d}{\arg \min }\left\|R_{j}(\tau)-u(d) \cdot R_{0}(\tau-v(d))\right\|_{1}
\end{aligned}
$$

We search the $d$ within a search range and select $\hat{d}_{j}$ which minimizes the cost function as the estimate of the thickness of $j^{\text {th }}$ wall.

The flow diagram of the proposed approach is depicted in Fig. 4.

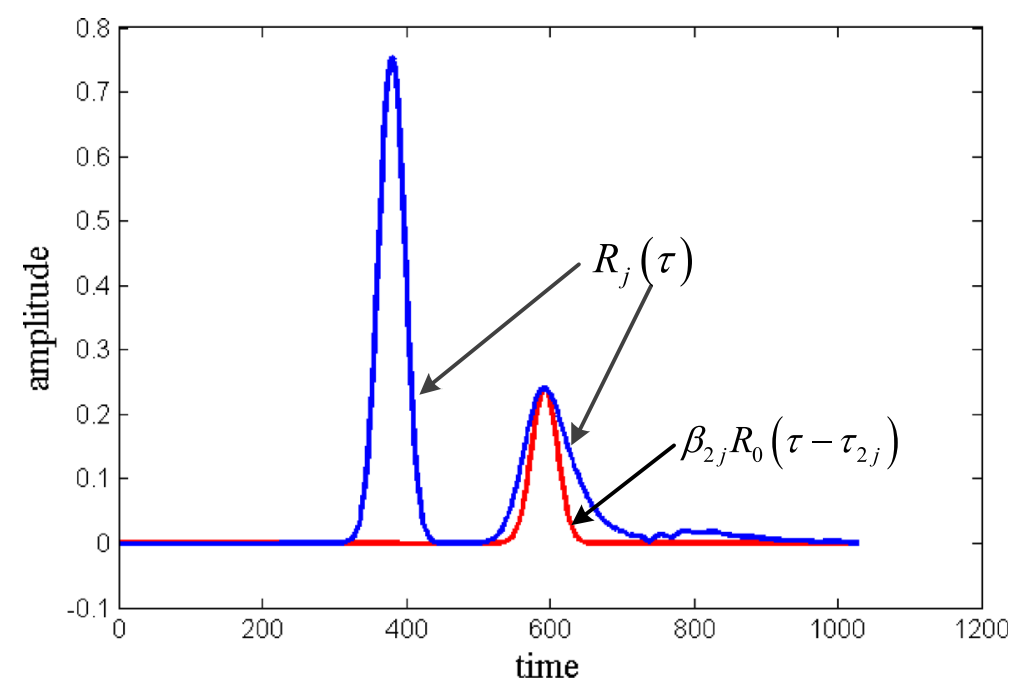

Fig. 3 An illustration of the cost function 


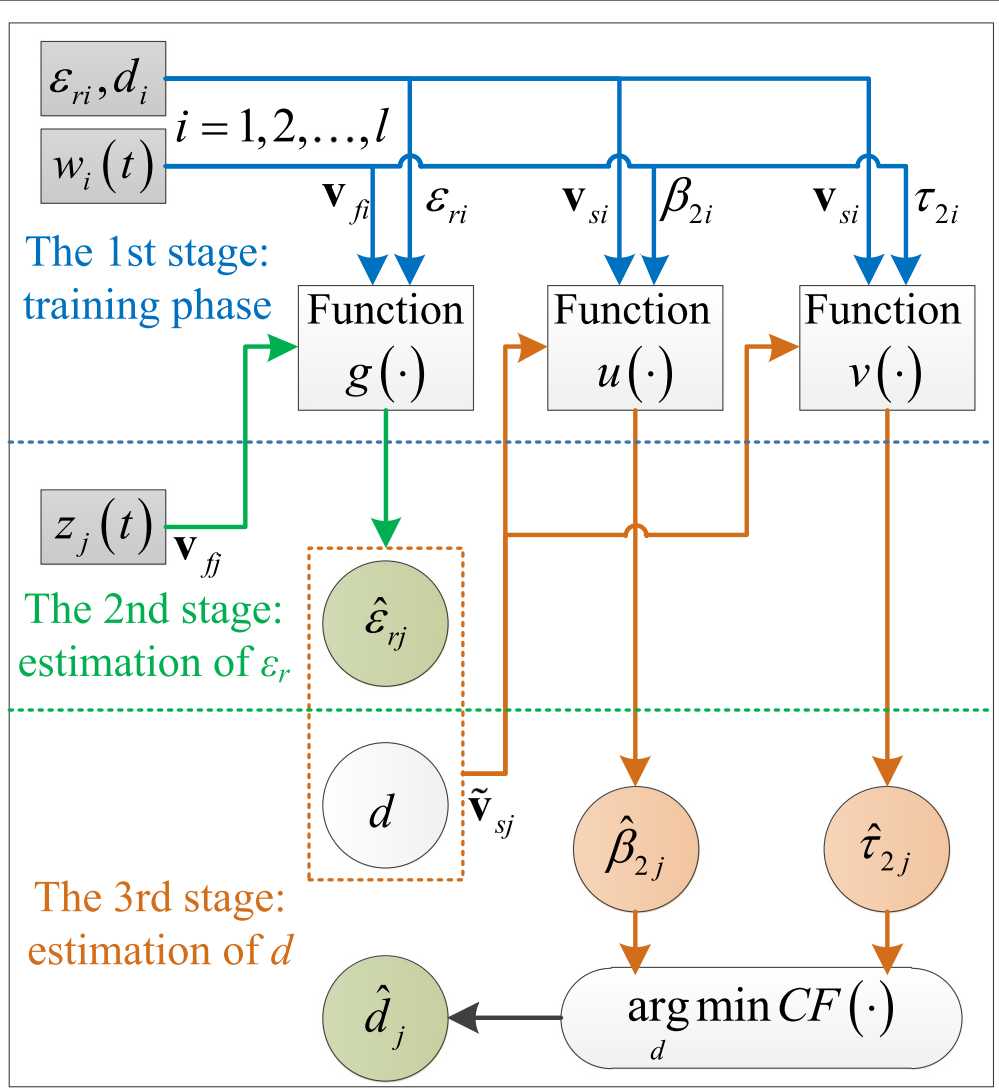

Fig. 4 Flow diagram of the proposed approach

\section{Simulation results}

\subsection{Description of the simulation}

The proposed approach and the regular method are both applied on the data that are generated using FDTD simulations. The settings in the simulation are listed as follows:

1. The transmitted radar signal is a Gaussian short-pulse with 1-GHz-frequency bandwidth. The carrier frequency is $1.5 \mathrm{GHz}$.

2. The transmitting and receiving antennas are placed near each other and both against the wall with a standoff distance of $1 \mathrm{~m}$.

3. Vertical polarization is adopted, and the incident angle is set to zero.

4. The conductivity of the wall is set at 0.01 , and the imaginary part of the permittivity of the wall is neglected [33].

5. The SVR part is accomplished using the LIBSVM software package [34], in which $\varepsilon$-SVR is adopted to perform the regression analysis. By comparing the performances of multiple kernel functions using cross validation on training set, radial basis function (RBF) is selected as the kernel function used in this simulation. The standard form of the RBF Kernel is $K(x, y)=\exp \left(-\gamma\|x-y\|^{2}\right)$.
6. The parameters $(C$ and $\gamma)$ that are needed in $\varepsilon$-SVR are estimated by the well-known $k$-fold cross validation in this simulation, with $k=4$. In $k$-fold cross-validation, the training data is randomly partitioned into $k$ equally sized subsets. Then in $i^{\text {th }}$ validation $i=(1,2, \ldots k)$, the regression function with the parameters $\left(C_{h}, \gamma_{o}\right)$ is built using $k-1$ subsets as training set. $h$ and $o$ are the $h^{\text {th }}$ and the $o^{\text {th }}$ elements of the parameters $C$ and $\gamma$. The performance is measured by the mean square error (MSE) on the output from the $i^{\text {th }}$ subset that is used as the test set. The procedure is repeated $k$ times, and the average of MSE is calculated. Through searching $C$ and $\gamma$ on a userdefined grid, the pair of parameters which provides the best average MSE will be chosen out [35].

\subsection{Primary results}

\section{Training data:}

For the training data, the permittivity of the wall is composed of 11 equispaced samples within the range of [4.0 - 14.0], and the thickness of the wall varies from 6.0 to $26.0 \mathrm{~cm}$ discretely with intervals of $2 \mathrm{~cm}$. Thus, for the training phase, there are a total of $\ell=121$ echo samples. There is no target in the scene when we collect the training data. 
2. Test data (no target):

For the test data, the set of permittivities and the thicknesses of the walls are composed of 11 equispaced samples within the ranges of [4.5-14.5] and [7.0-27.0 cm], respectively. Thus for the test phase, there are also 121 samples in test set. For the $j^{t h}$ test sample, the permittivity and thickness of the corresponding wall are denoted as $\varepsilon_{r j}$ and $d_{j}$, respectively.

3. Test data (target with $R=10 \mathrm{~cm}, D_{y j}=d_{j}$ ):

In order to test the robustness of the proposed approach when targets exist behind the wall, we generate another test data set, in which the data are collected with the presence of a metal cylinder behind the wall. The cylinder with radius of $10 \mathrm{~cm}$ is placed away from the wall with a distance $D_{y j}$ that is equal to the current wall thickness $d_{j}$ in each sample. Here, $D_{y j}$ is defined as the distance between the target's front surface and the wall's rear surface in the $j^{\text {th }}$ test sample.

For the proposed SVR method, the training phase and the estimation procedure are carried out in sequence as described above. The pairs of parameters for the three regression functions are obtained by the $k-$ fold method, and they are $\left(C=1180, \gamma=3.05 \times 10^{-5}\right)$ for function $g(\cdot),(C=7130, \gamma=0.0068)$ for $u(\cdot)$, and $(C=$ 65536, $\gamma=0.0013)$ for $v(\cdot)$. The search range of $d$ is from 5 to $30 \mathrm{~cm}$ with step size of $1 \mathrm{~cm}$ throughout the simulation. The simulation results show that such a precision of $1 \mathrm{~cm}$ is a good tradeoff between the estimation performance and the computational complexity.

At each run, the proposed approach estimates the permittivity and the thickness of the wall for the current test sample. The estimation results of the proposed approach for the samples in training set, the test set without target and the test set with target, are shown in Fig. 5a, b, c, respectively. In these figures as well as the following illustrations, the solid lines represent the true permittivities while the circle markers represent the estimated permittivities; meanwhile, the dashed lines represent the true thicknesses while the star markers represent the estimated thicknesses.

It is seen that the proposed approach is able to provide the estimates of wall parameters which approximate to the true values, even with the presence of the target. We notice that when the target exists, the estimated thicknesses of those samples which are with large permittivities and thickness deviate from their true values a little. This is because when the wall has large permittivity and thickness, the echo from the rear side of the wall will be attenuated and distorted seriously, which means that the echo will have a small amplitude and a long span of time, and thus tends to be more affected by the target echo.

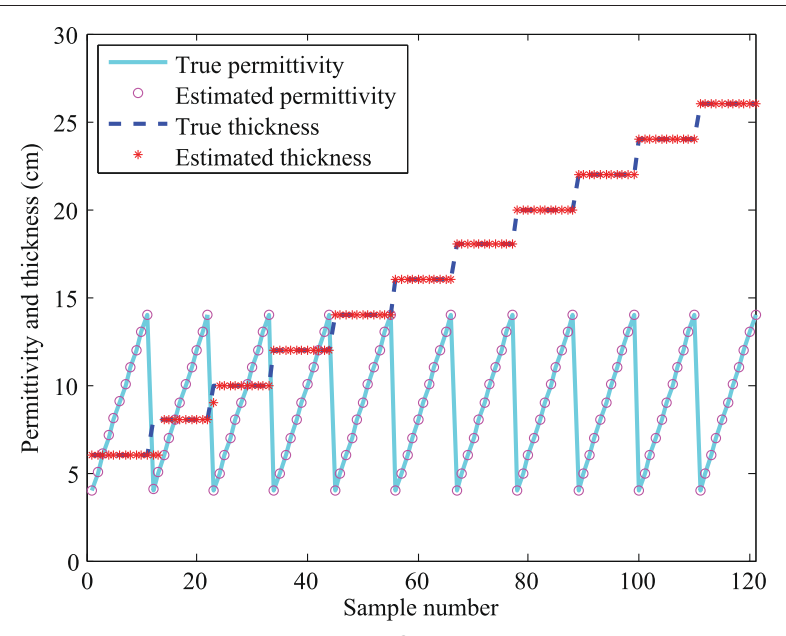

a

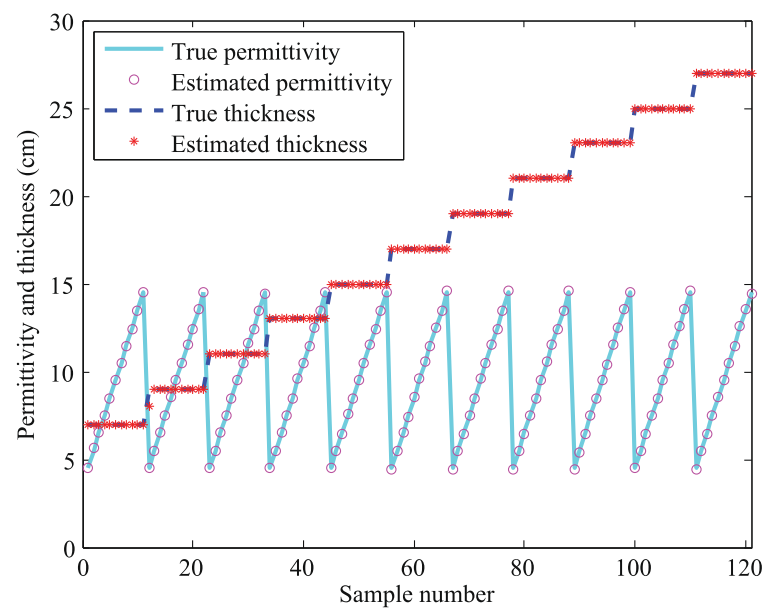

b

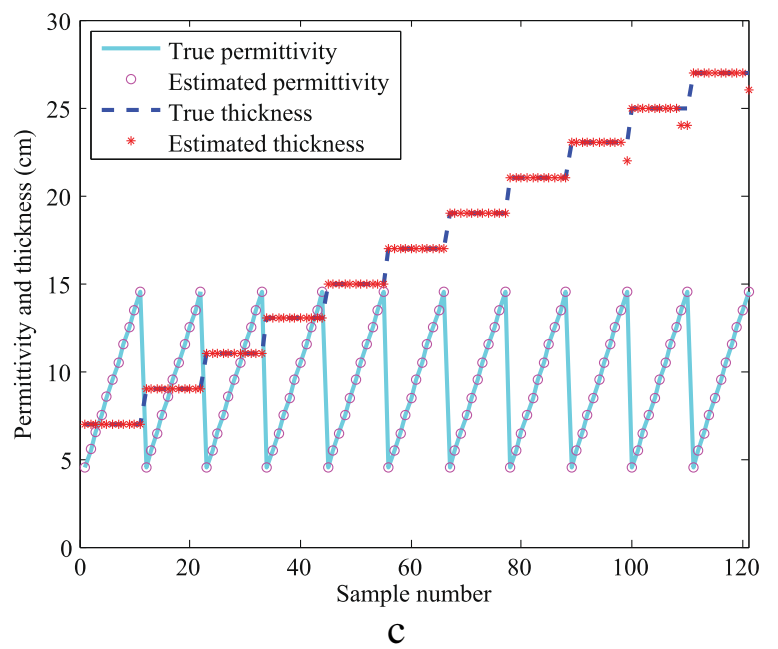

Fig. 5 Simulation results obtained by the proposed approach. a Training data; $\mathbf{b}$ test data (no targets); $\mathbf{c}$ test data (target with $R=10$ $\mathrm{cm}, D_{y j}=d_{j}$ ) 
For the purpose of comparison, we apply the regular SVR method on those data sets. The pairs of parameters for the two regression functions are $(C=3.03, \gamma=$ $\left.9.76 \times 10^{-4}\right)$ for the regression function of permittivity and $(C=73.5, \gamma=0.0118)$ for the function of thickness. The estimation results are shown in Fig. 6 . We see that in Fig. 6a, the estimated results are acceptable, but in Fig. 6b, the test results are not as good as that on the training set. In Fig. 6c, the performance of the regular SVR method deteriorates rapidly since the presence of the target makes the test data different from the training data dramatically.

\subsection{Further results}

In order to inspect the effect of the presence of the target carefully, more test sets are generated in different scenarios and used to measure the performance of the proposed approach.

It is obvious that the closer the target is to the wall, the more seriously the wall echoes will be interfered with the target echo. Hence, we change the distance between the target and the wall in descending order to see the variation of the estimation result. Three test data sets are generated under the situations in which the size of the target is fixed but the the distance $D_{y j}$ is set to be 1) three times the current wall thickness for each sample in the first data set; 2) twice the current wall thickness for each sample in the second data set; and 3) $1 \mathrm{~cm}$ in the third data set, respectively.

The simulation results based upon these data sets are shown in Fig. 7. As we expected, the estimation results are improved as the target moves far away from the wall. It is seen that in Fig. 7a, b, the estimation results are quite accurate. In Fig. 7c, since the target is very close to the wall, some estimated values of the thickness are larger than the true values. But the errors are relatively small and can be tolerated. In comparison, the estimation results of the regular SVR method is shown in Fig. 8. Similarly, the trend of the performances from Fig. $8 \mathrm{a}-\mathrm{c}$ also conform to our analysis. However, the performances of the regular SVR method are poorer than that of the proposed approach, especially when the target stands very close to the wall, such as in Fig. 8c. The estimated values of the thickness in it are almost totally wrong.

Next, the size of the target is changed in increasing order since it is predictable that a stronger echo from a bigger target will have more serious interference on the echo from the wall. Therefore, three more test data sets are generated under three different scenarios, wherein the distance $D_{y j}$ is fixed, but the radius of the target is set to be 1) $2 \mathrm{~cm}$ for the first data set; 2) $5 \mathrm{~cm}$ for the second data set, and 3) $20 \mathrm{~cm}$ for the third data set. The estimated results from these data sets provided by the proposed approach are shown in Fig. 9, which are consistent with our expectation. In Fig. 9c, the estimation errors of

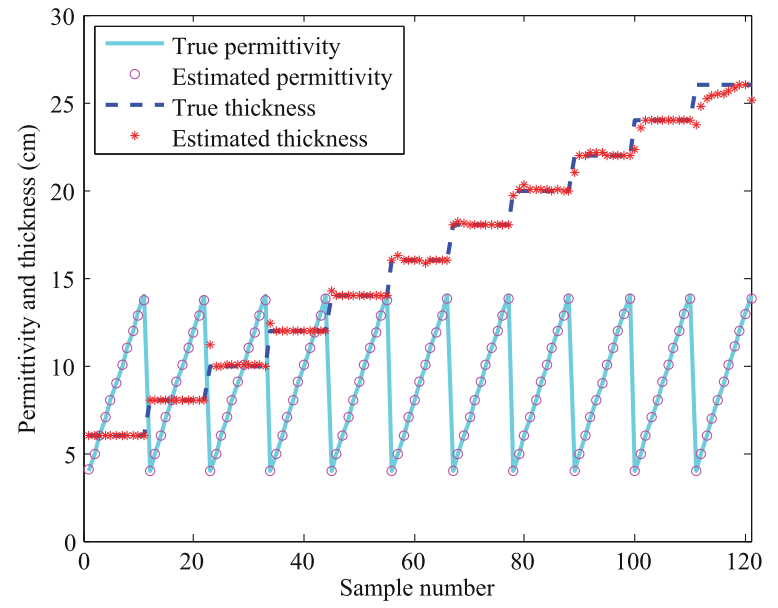

a

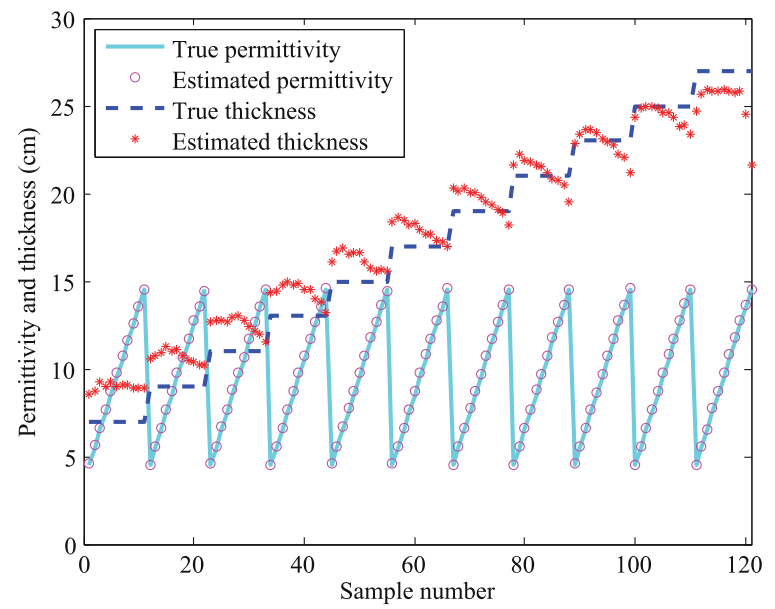

b

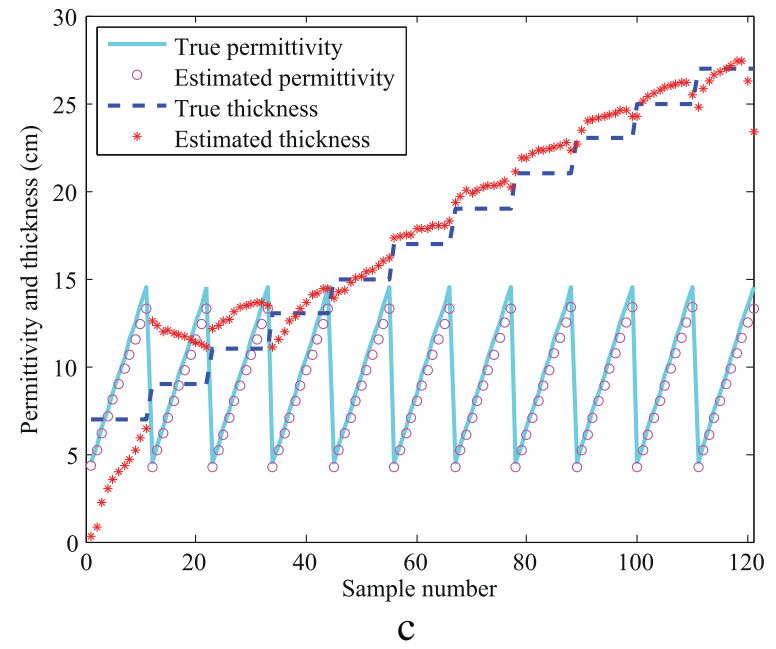

Fig. 6 Simulation results obtained by the regular SVR method. a Training data; b test data (no targets); c test data (target with $R=10$ $\left.\mathrm{cm}, D_{y j}=d_{j}\right)$ 

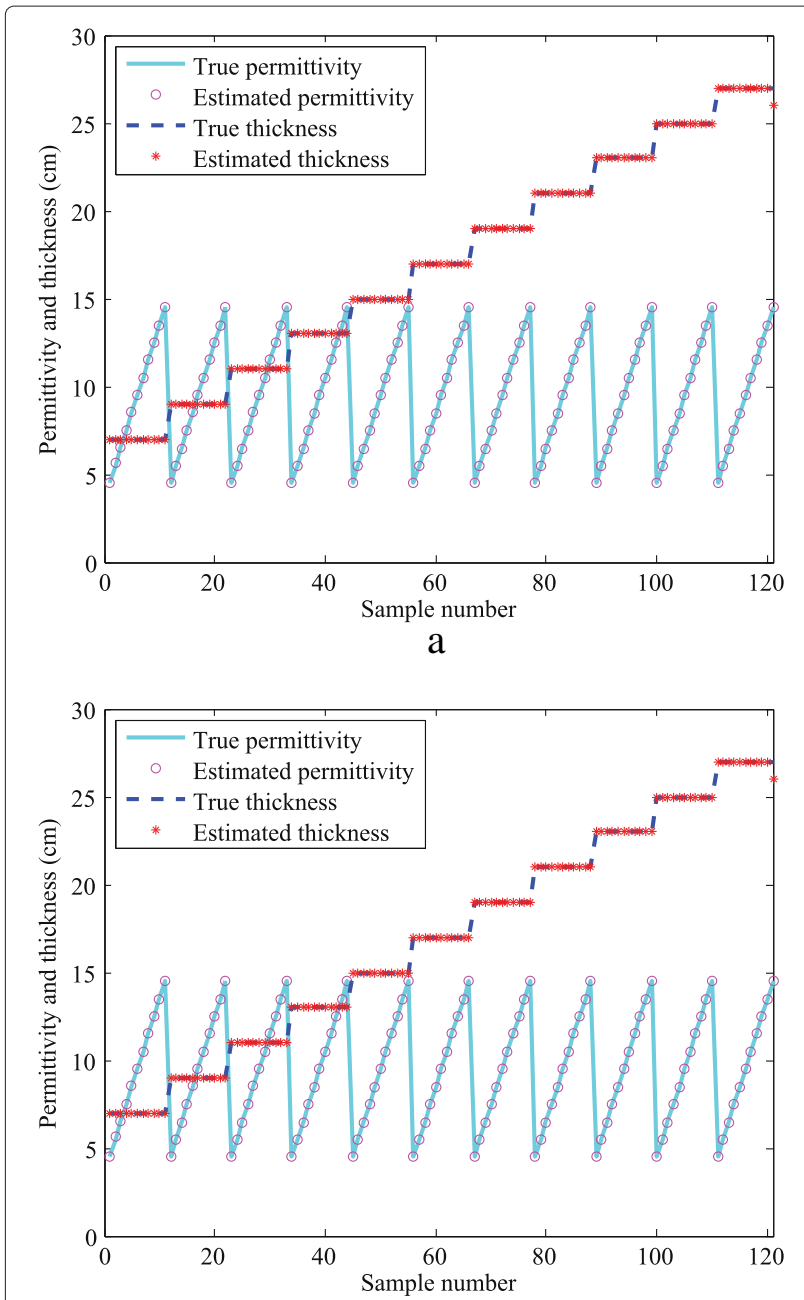

b

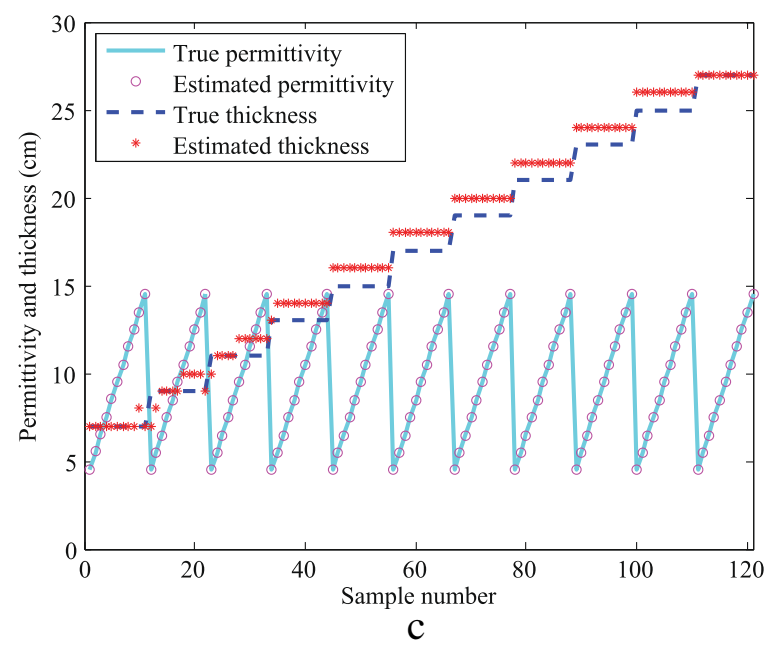

Fig. 7 Performances of the proposed approach as the standoff distance changes. a Test data $\left(R=10 \mathrm{~cm}, D_{y j}=3 \times d_{j}\right) ; \mathbf{b}$ test data $\left(R=10 \mathrm{~cm}, D_{y j}=2 \times d_{j}\right) ; \mathbf{c}$ test data $\left(R=10 \mathrm{~cm}, D_{y j}=1 \mathrm{~cm}\right)$

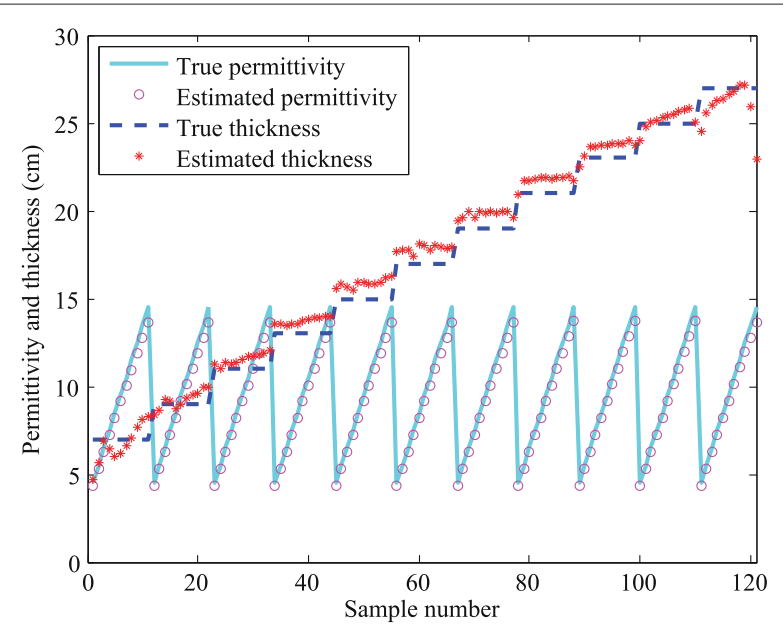

a

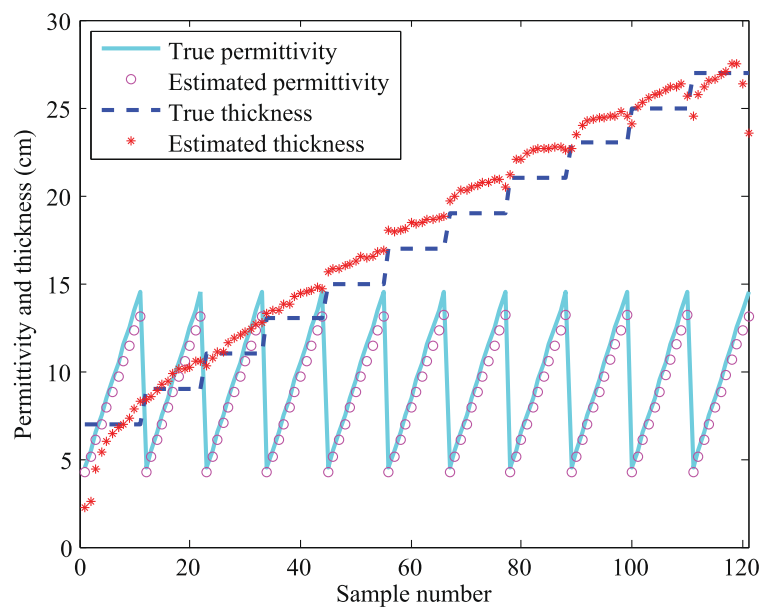

b

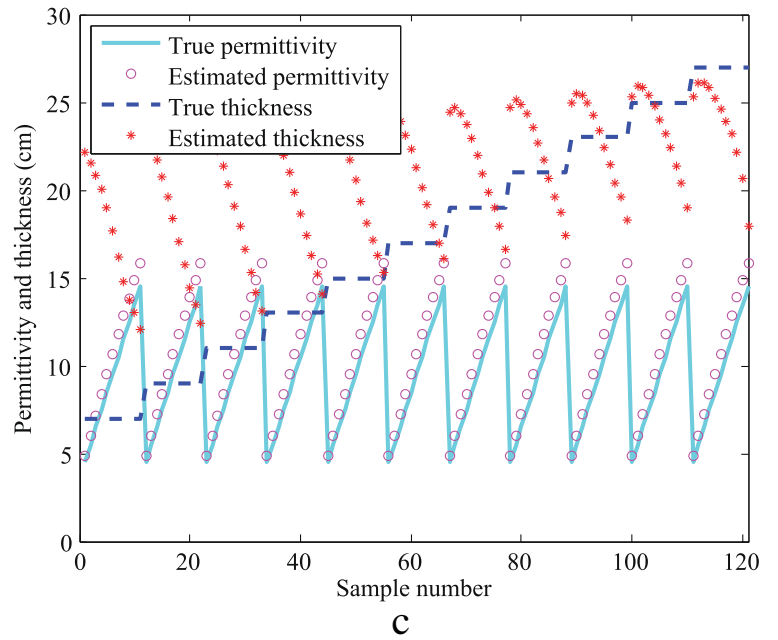

Fig. 8 Performances of the regular SVR method as the standoff distance changes. a Test data $\left(R=10 \mathrm{~cm}, D_{y j}=3 \times d_{j}\right)$; b test data $\left(R=10 \mathrm{~cm}, D_{y j}=2 \times d_{j}\right) ; \mathbf{c}$ test data $\left(R=10 \mathrm{~cm}, D_{y j}=1 \mathrm{~cm}\right)$ 


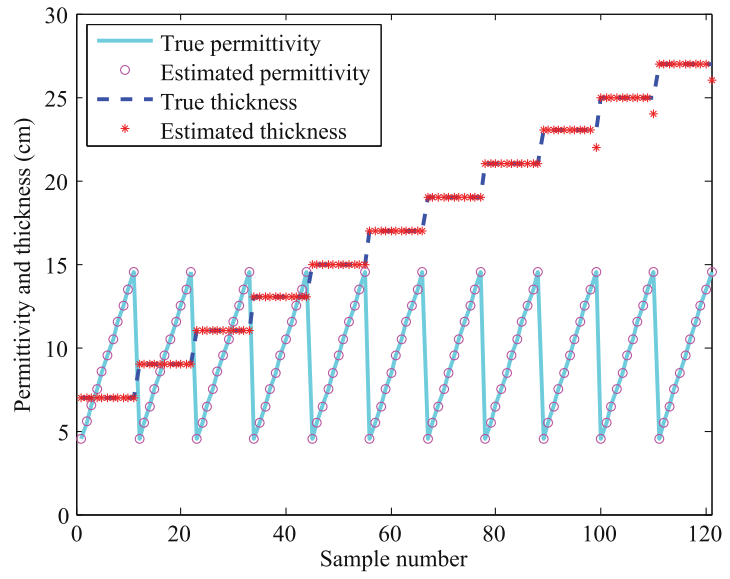

a

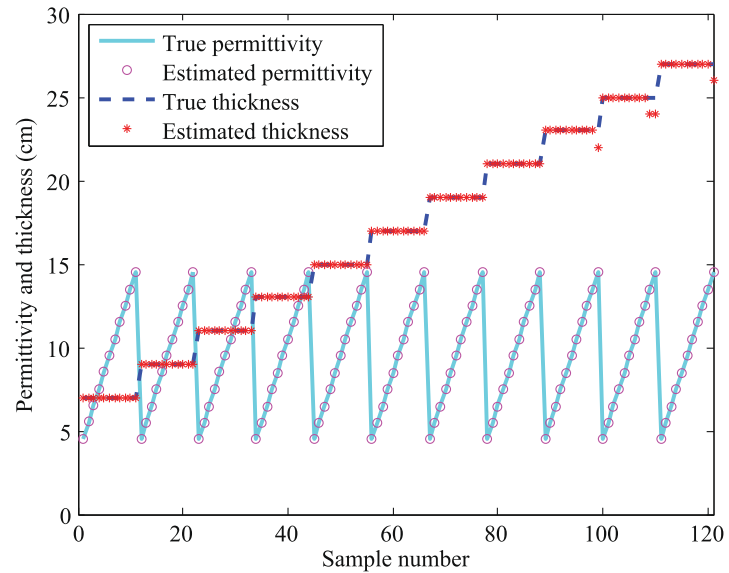

b

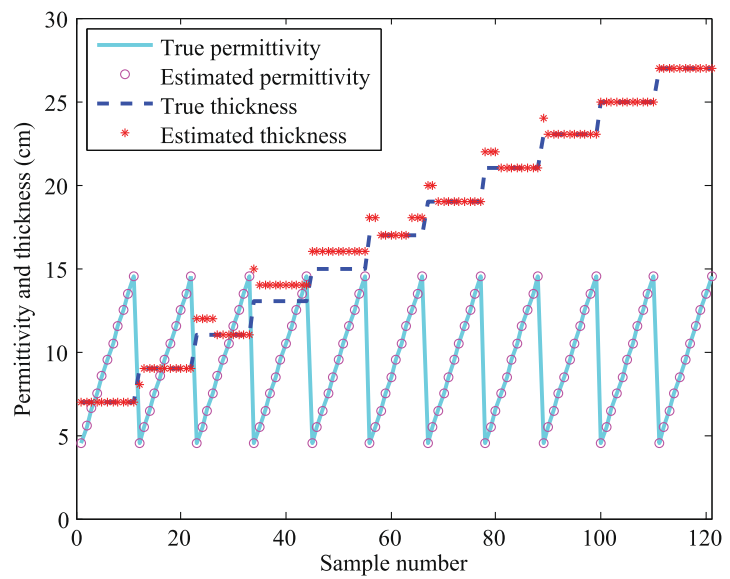

C

Fig. 9 Performances of the proposed approach as the size of the target changes. a Test data $\left(R=2 \mathrm{~cm}, D_{y j}=d_{j}\right)$; b test data $(R=5 \mathrm{~cm}$, $\left.D_{y j}=d_{j}\right) ; \mathbf{c}$ test data $\left(R=20 \mathrm{~cm}, D_{y j}=d_{j}\right)$

the thickness are larger than those in Fig. 9a, b, but it is still acceptable. The results obtained by the regular SVR method are given in Fig. 10 for contrast. The performance

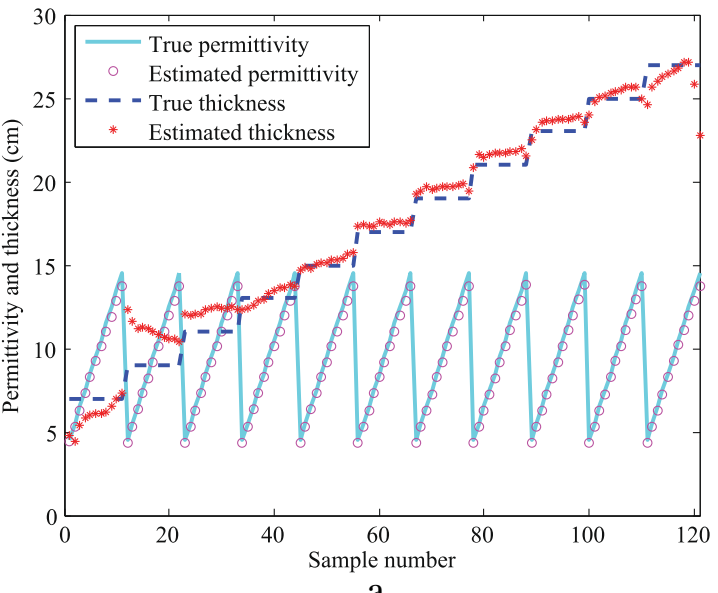

a

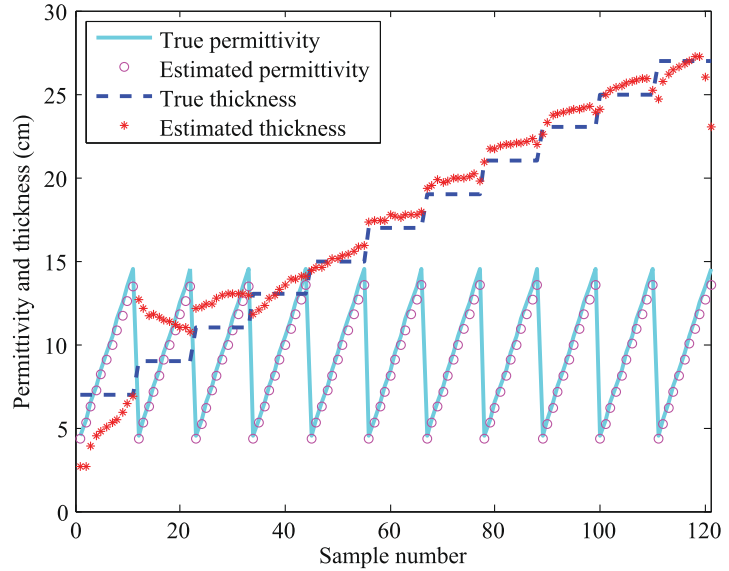

b

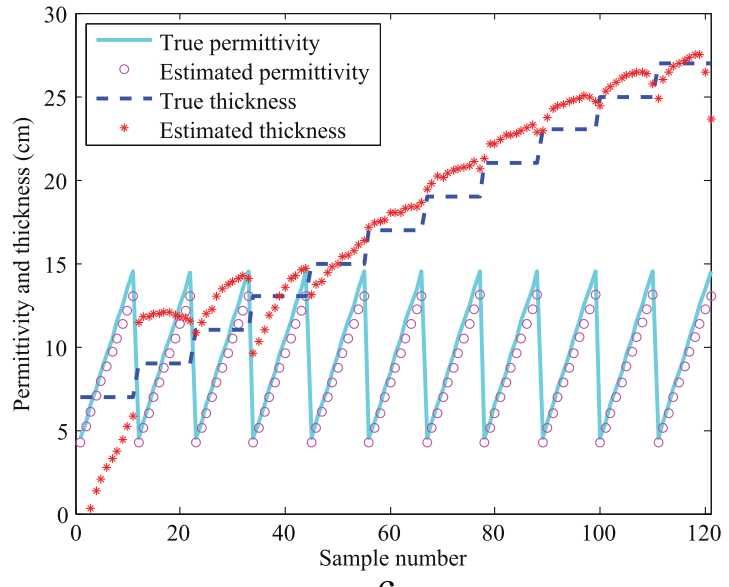

c

Fig. 10 Performances of the regular SVR method as the size of the target changes. a Test data $\left(R=2 \mathrm{~cm}, D_{y j}=d_{j}\right) ; \mathbf{b}$ Test data $(R=5 \mathrm{~cm}$, $\left.D_{y j}=d_{j}\right) ; \mathbf{c}$ Test data $\left(R=20 \mathrm{~cm}, D_{y j}=d_{j}\right)$

of the regular SVR method is still poorer than that of the proposed approach, especially in Fig. 10c due to the large size of the target. 
Table 1 RMSEs of the estimates

\begin{tabular}{|c|c|c|c|c|c|c|c|c|c|c|}
\hline RMSE & Train $^{b}$ & Test $^{b}$ & $20 a^{c}$ & $20 b^{c}$ & $20 c$ & $20 d$ & $10 a$ & $10 b$ & $10 c$ & $10 d$ \\
\hline$[\mathrm{A}] \varepsilon_{r}{ }^{\mathrm{a}}$ & 0.03 & 0.05 & 0.02 & 0.02 & 0.02 & 0.02 & 0.02 & 0.02 & 0.02 & 0.02 \\
\hline$[\mathrm{A}] d^{\mathrm{a}}$ & 0.27 & 0.09 & 1.25 & 0.58 & 0.16 & 0.09 & 0.88 & 0.18 & 0.09 & 0.09 \\
\hline$[\mathrm{B}] \varepsilon_{r}{ }^{\mathrm{a}}$ & 0.08 & 0.20 & 1.45 & 0.87 & 1.01 & 0.56 & 1.09 & 0.70 & 0.80 & 0.46 \\
\hline \multirow[t]{6}{*}[B]{$d$} & 0.35 & 1.40 & 7.03 & 2.29 & 1.85 & 1.13 & 6.38 & 1.82 & 1.39 & 0.88 \\
\hline & & & $5 a$ & $5 b$ & $5 c$ & $5 d$ & $2 a$ & $2 b$ & $2 c$ & $2 d$ \\
\hline & & & 0.02 & 0.02 & 0.02 & 0.02 & 0.02 & 0.02 & 0.02 & 0.02 \\
\hline & & & 0.33 & 0.18 & 0.09 & 0.09 & 0 & 0.16 & 0.09 & 0.09 \\
\hline & & & 0.71 & 0.55 & 0.69 & 0.43 & 0.30 & 0.41 & 0.57 & 0.41 \\
\hline & & & 5.09 & 1.43 & 1.16 & 0.80 & 3.41 & 1.08 & 0.96 & 0.77 \\
\hline
\end{tabular}

\footnotetext{
a $\left[\right.$ A] represents the new proposed approach. [B] represents the regular SVR method. Permittivity and thickness are denoted as $\varepsilon_{r}$ and $d$, respectively

b "Train" refers to the training data. "Test" refers to the test data (with no target)

" $20 \mathrm{a}$ " represents the test data in which the number " 20 " indicates that the radius of the target is $20 \mathrm{~cm}$ and the letter "a" refers to the distance between the target and the wall. Here, "a" $=1 \mathrm{~cm} " \mathrm{~b} "=1 \times d_{j}$. "c" $=2 \times d_{j} . " d "=3 \times d_{j}$
}

The root-mean-square errors (RMSEs) of the estimated results that can be used to evaluate the performances of the estimation methods in detail are given in Table 1. In addition to the data sets used before, some more data sets are generated and tested, and their estimation results are listed in the table. These simulation results confirm again that the proposed approach can provide satisfactory estimation performance for WPE and prove that the proposed approach is superior than the regular SVR method in robustness, accuracy and efficiency.

\section{Conclusion}

In this paper, SVR is introduced into WPE for the first time. But unlike the typical regression problem, the test data in WPE, i.e., the received radar returns from the walls, are interfered with the target echoes heavily. Against this difficulty, a new approach combining SVR and an optimization procedure is proposed, in which the permittivity is estimated by SVR firstly, and then the thickness is achieved by minimizing the cost function based on the estimated permittivity and the instrumental variables provided by SVR also. The numerical experiment results show that the proposed approach can provide accurate estimates of the wall parameters in diverse contexts and outperforms the regular SVR method. In addition, the proposed method has higher computational efficiency since the input feature vectors are in a lower dimension. The training samples needed by this approach can be achieved in advance, thus it is feasible to apply the proposed approach in practice.

\section{Competing interests}

The authors declare that they have no competing interests.

\section{Acknowledgements}

The work in this paper is supported in part by the General Programs of National Natural Science Foundation of China under Grant No. 61172155 and under Grant No. 61331020.
Received: 25 November 2014 Accepted: 25 June 2015

Published online: 08 July 2015

\section{References}

1. F Ahmad, MG Amin, SA Kassam, Synthetic aperture beamformer for imaging through a dielectric wall. Aerosp. Electron. Syst. IEEE Trans. 41(1), 271-283 (2005)

2. B Chen, $T$ Jin, B Lu, Z Zhou, Building interior layout reconstruction from through-the-wall radar image using MST-based method. Eurasip J Adv Signal Process. 2014(1), 1-9 (2014)

3. C Clemente, A Balleri, K Woodbridge, JJ Soraghan, Developments in target micro-doppler signatures analysis: Radar imaging, ultrasound and through-the-wall radar. Eurasip J. Adv. Signal Process. 2013(1), 1-18 (2013)

4. F Ahmad, MG Amin, T Dogaru, Partially sparse imaging of stationary indoor scenes. Eurasip J. Adv. Signal Process. 2014(1), 1-15 (2014)

5. M Dehmollaian, M Thiel, K Sarabandi, Through-the-wall imaging using differential SAR. Geoscience Remote Sensing IEEE Trans. 47(5), 1289-1296 (2009)

6. T Dogaru, C Le, SAR images of rooms and buildings based on FDTD computer models. Geoscience Remote Sensing IEEE Trans. 47(5), 1388-1401 (2009)

7. Y-Z Hu, T-J Li, Z-O Zhou, Use of the location inverse solution to reduce ghost images. Eurasip J. Adv. Signal Process. 2010, 1-12 (2010)

8. M Dehmollaian, K Sarabandi, Refocusing through building walls using synthetic aperture radar. Geoscience Remote Sensing IEEE Trans. 46(6), 1589-1599 (2008)

9. R Solimene, FS Prisco, R Pierri, Three-dimensional through-wall imaging under ambiguous wall parameters. Geoscience Remote Sensing IEEE Trans. 47(5), 1310-1317 (2009)

10. W Genyuan, MG Amin, Imaging through unknown walls using different standoff distances. Signal Process. IEEE Trans. 54(10), 4015-4025 (2006)

11. P Protiva, J Mrkvica, J MacHac, Estimation of wall parameters from time-delay-only through-wall radar measurements. IEEE Trans. Antennas Propagation. 59(11), 4268-4278 (2011)

12. F Ahmad, MG Amin, G Mandapati, Autofocusing of through-the-wall radar imagery under unknown wall characteristics. Image Process. IEEE Trans. 16(7), 1785-1795 (2007)

13. X Chen, W Chen, in Antennas and Propagation Society International Symposium (APSURSI), IEEE. A simple method for image autofocusing in through-wall radar imaging (IEEE Piscataway, New Jersey, 2013), pp. 530-531

14. $X L i, X$ Huang, $T$ Jin, Estimation of wall parameters by exploiting correlation of echoes in time domain. Electron. Lett. 46(23), 1563-1565 (2010)

15. S Fukuda, H Hirosawa, in Geoscience and Remote Sensing Symposium, IGARSS '01. IEEE International. Support vector machine classification of land cover: application to polarimetric SAR data, vol. 1 (IEEE Piscataway, New Jersey, 2001), pp. 187-1891 
16. Z Qun, JC Principe, Support vector machines for SAR automatic target recognition. Aerospace Electron. Syst. IEEE Trans. 37(2), 643-654 (2001)

17. S Kent, NG Kasapoglu, M Kartal, in Radar Conference. RADAR '08. IEEE. Radar target classification based on support vector machines and high resolution range profiles (IEEE Piscataway, New Jersey, 2008), pp. 1-6

18. E Pasolli, F Melgani, M Donelli, Automatic analysis of GPR images: A pattern-recognition approach. Geoscience Remote Sensing IEEE Trans. 47(7), 2206-2217 (2009)

19. T Yajuan, L Xiapu, Y Zijie, in Machine Learning for Signal Processing. Proceedings of the 14th IEEE Signal Processing Society Workshop. Ocean clutter suppression using one-class SVM (IEEE Piscataway, New Jersey, 2004), pp. 559-568

20. K Youngwook, L Hao, Human activity classification based on micro-doppler signatures using a support vector machine. Geoscience Remote Sensing IEEE Trans. 47(5), 1328-1337 (2009)

21. W Chun-Hsin, H Jan-Ming, DT Lee, Travel-time prediction with support vector regression. Int. Transport. Syst. IEEE Trans. 5(4), 276-281 (2004)

22. D Singh, in Geoscience and Remote Sensing Symposium, IGARSS '07. IEEE International. An efficient electromagnetic approach to train the SVM for depth estimation of shallow buried objects with microwave remote sensing data (IEEE Piscataway, New Jersey, 2007), pp. 4961-4964

23. CL Bastard, V Baltazart, X Derobert, W Yide, in Ground Penetrating Radar (GPR), 14th International Conference On. Support vector regression method applied to thin pavement thickness estimation by GPR (IEEE Piscataway, New Jersey, 2012), pp. 349-353

24. CL Bastard, W Yide, V Baltazart, X Derobert, Time delay and permittivity estimation by ground-penetrating radar with support vector regression. Geoscience Remote Sensing Lett. IEEE. 11(4), 873-877 (2014)

25. LM Frazier, in Enabling Technologies for Law Enforcement and Security. Radar surveillance through solid materials (International Society for Optics and Photonics Bellingham, Washington USA, 1997), pp. 139-146

26. TB Gibson, DC Jenn, Prediction and measurement of wall insertion loss. Antennas Propagation IEEE Trans. 47(1), 55-57 (1999)

27. JD Jackson, Classical Electrodynamics, 3rd Edition. (Wiley, New York, 1999)

28. MG Amin, Through-the-Wall Radar Imaging. (CRC Press, Boca Raton, Florida, 2010)

29. V Vapnik, The Nature of Statistical Learning Theory. (Springer, New York, 1995)

30. H Drucker, CJ Burges, L Kaufman, A Smola, V Vapnik, Support vector regression machines. Adv. Neural Inf. Process. Syst. 9, 155-161 (1997)

31. A Smola, B Schölkopf, A tutorial on support vector regression. Stat. Comput. 14(3), 199-222 (2004)

32. V Cherkassky, Y Ma, Practical selection of svm parameters and noise estimation for svm regression. Neural Netw. 17(1), 113-126 (2004)

33. A Muqaibel, A Safaai-Jazi, A Bayram, AM Attiya, SM Riad, Ultrawideband through-the-wall propagation. Microwaves Antennas Propagation IEEE Proc. 152(6), 581-588 (2005)

34. C-C Chang, C-J Lin, LIBSVM: A library for support vector machines. ACM Trans. Intell. Syst. Technol. 2(3), 1-27 (2011)

35. B Schölkopf, AJ Smola, RC Williamson, PL Bartlett, New support vector algorithms. Neural Comput. 12(5), 1207-1245 (2000)

\section{Submit your manuscript to a SpringerOpen ${ }^{\circ}$ journal and benefit from:}

- Convenient online submission

- Rigorous peer review

- Immediate publication on acceptance

- Open access: articles freely available online

- High visibility within the field

- Retaining the copyright to your article

Submit your next manuscript at $\boldsymbol{\nabla}$ springeropen.com 